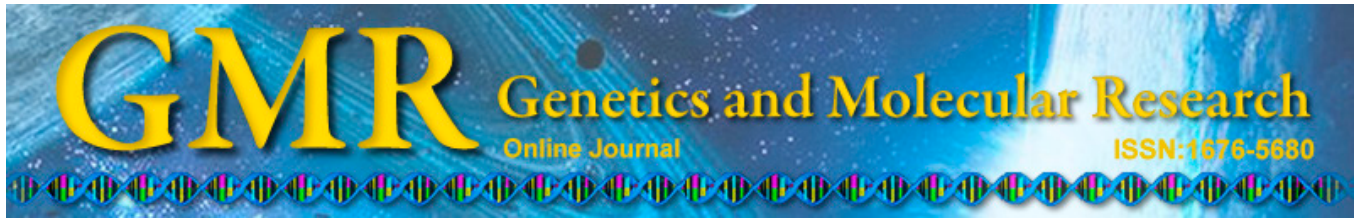

\title{
Effect of ulinastatin on HMGB1 expression in rats with acute lung injury induced by sepsis
}

\author{
S.Y. Wang ${ }^{1 *}$, Z.J. Li ${ }^{2 *}$, X. Wang ${ }^{1}$, W.F. Li ${ }^{1}$ and Z.F. $\operatorname{Lin}^{1}$ \\ ${ }^{1}$ Department of Emergency and Critical Care Medicine, \\ Shanghai Changzheng Hospital, The Second Military Medical University, \\ Shanghai, China \\ ${ }^{2}$ Department of Anesthesiology, Shanghai Changzheng Hospital, \\ The Second Military Medical University, Shanghai, China \\ *These authors contributed equally to this study. \\ Corresponding authors: W.F. Li / Z.F. Lin \\ E-mail: liwenfang201441@126.com / linzhaofen@sina.com
}

Genet. Mol. Res. 14 (2): 4344-4353 (2015)

Received May 14, 2014

Accepted November 3, 2014

Published April 30, 2015

DOI http://dx.doi.org/10.4238/2015.April.30.7

\begin{abstract}
The aim of this study was to investigate the influence of ulinastatin (UTI) on high mobility group box 1 (HMGB1), tumor necrosis factor (TNF)- $\alpha$, and interleukin (IL)- 6 expression in acute lung injury (ALI) rats with sepsis caused by cecal ligation and puncture (CLP) surgery, as well as to examine the underlying biological mechanism. Thirty rats were randomly and evenly divided into sham (control), CLP, and CLP + UTI groups. Thirty minutes after the surgery, the rats in the CLP + UTI group received UTI via the caudal vein, while normal saline was administered to rats in the other groups. Blood, lung tissues, and bronchoalveolar lavage fluid (BALF) were collected at different time points $(6,12,24$, and $48 \mathrm{~h})$ after surgery for determination of related indicators. Compared with the CLP group, rats in the CLP + UTI group exhibited higher seven day survival rates, less lung injury, and decreased HMGB1 expression in the lung tissue, serum, and BALF. In addition, the levels of TNF- $\alpha$ and IL- 6 at $24 \mathrm{~h}$ in the CLP + UTI group were markedly lower than those in the CLP group. These results suggest that
\end{abstract}


by deregulation, UTI might decrease the lung injury and increase the survival time of ALI rats by downregulating HMGB1 expression as well as by inhibiting TNF- $\alpha$ and IL-6 levels in serum and BALF.

Key words: Acute lung injury; Ulinastatin; High mobility group box 1; Tumor necrosis factor $\alpha$; Interleukin-6

\section{INTRODUCTION}

Acute lung injury (ALI), clinically characterized by respiratory distress and intractable hypoxemia, is a common clinical syndrome secondary to severe trauma, infection, shock, and major surgery. ALI is associated with considerable morbidity and mortality. It is estimated that approximately 200,000 patients develop ALI per year in the United States, leading to 75,000 deaths (Rubenfeld et al., 2005). Even though significant advances have occurred in the management of ALI, the mortality remains unacceptably high, ranging between 34 and $68 \%$ (Ware and Matthay, 2000). Thus, it is urgently necessary to explore new therapies to improve the survival outcomes of patients with ALI.

Ulinastatin, also known as urinary trypsin inhibitor (UTI) and Kunitz-type proteinase inhibitor, is an acidic glycoprotein extracted from fresh urine and blood of healthy adult men (Sumi et al., 1977; Hochstrasser et al., 1981). It is reported that in addition to inhibiting plasmin, trypsin, chymotrypsin, elastase, and various pancreatic enzymes (Nishiyama et al., 1996), UTI also suppresses activation of inflammatory cells (e.g., neutrophils and leukocytes) and the production of inflammatory mediators such as tumor necrosis factor (TNF)- $\alpha$, and interleukin (IL)-1 $\beta$, IL-6, IL-8, and IL-15 (Ito et al., 2005; Fang et al., 2011; Cao et al., 2012; Shen et al., 2012; Wang et al., 2013). Therefore, UTI is suggested to be a potential therapeutic agent for ALI (Ito et al., 2005; Fang et al., 2011; Shen et al., 2012; Wang et al., 2013). However, the mechanism of UTI treatment of ALI has not been fully elucidated.

High mobility group box 1 (HMGB1) is one of the central factors for starting and maintaining the cascade amplification of inflammation, and thus plays crucial roles in many diseases, including ALI (Silva et al., 2007). Inhibition of HMGB1 expression may represent an underlying approach for preventing or minimizing ALI (Hidaka et al, 2011; Entezari et al., 2014). A recent study demonstrated that UTI preconditioning lowered HMGB1 expression and attenuated the inflammatory reaction of hepatic ischemia reperfusion injury in rats (Tong et al., 2014). However, whether UTI also inhibits HMGB1 in ALI remains unclear. In this study, we employed an animal model of cecal ligation and perforation (CLP) to assess the possible protective mechanism of UTI for CLP-induced ALI by detecting the expression of HMGB1 and other inflammatory factors.

\section{MATERIAL AND METHODS}

\section{Animal grouping and treatment}

A total of 120 specific pathogen-free-derived male Sprague-Dawley rats (6-8 weeks of age, 180-200 g) were purchased from the Animal Center of the Secondary Military Medicine University (Shanghai, China, license No. SCXK-Shanghai 2012-0003). The rats were randomly divided into sham-operated (control, $\mathrm{N}=20)$, CLP $(\mathrm{N}=50)$, and CLP + UTI groups ( 
$=50$ ). CLP surgery was performed, and after $30 \mathrm{~min}$, the rats in the CLP + UTI group received intravenous injection of UTI $\left(1 \times 10^{5} \mathrm{U} / \mathrm{kg}\right)$ in their tails, while the rats in the other two groups were injected with normal saline. Ten rats in each group were used to observe the seven-day survival rate. At $6,12,24$, and $48 \mathrm{~h}$ after surgery, ten rats in each of the CLP and CLP + UTI groups were used for blood and lung tissue collection for further analyses. All animal experiments were approved by the institutional Animal Care and Use Committee and conducted according to the institutional guidelines for the care and use of laboratory animals.

\section{CLP surgery}

After a one-week acclimation period in the laboratory, the rats were fasted for $12 \mathrm{~h}$ but allowed water ad libitum. Sepsis was induced through CLP in rats, as previously described (Razavi et al., 2002). Briefly, rats were anesthetized via intraperitoneal injection of pentobarbital $(40 \mathrm{mg} / \mathrm{kg}$ ) and then shaved, and washed with $10 \%$ povidone-iodine in a supine position. A 2-cm midline incision was made on the anterior abdomen, and the cecum was exposed and ligated with a 3-O silk suture below the ileocecal valve. Sequentially, punctures were made in the cecum using a 20-gauge needle to induce sepsis. A small amount of stool was extruded from the puncture site, and the cecum was then returned to the peritoneum. The abdomen was closed with a 4-O chromic gut suture for the peritoneum and surgical staples for the skin. All animals received saline $(10 \mathrm{~mL} / \mathrm{kg})$ subcutaneously immediately after the surgery for anti-shock. Sham-operated animals underwent the same procedure with the exception that the cecum was neither ligated nor punctured. The seven-day survival rate was recorded after the surgery.

\section{Sample collection}

Blood samples $(2 \mathrm{~mL})$ were collected at the defined time points from postcava, centrifuged for $30 \mathrm{~min}$ at $4^{\circ} \mathrm{C}, 3000 \mathrm{rpm}$, and stored at $-80^{\circ} \mathrm{C}$ for further experiments. The rats were sacrificed by transection of the abdominal aorta and the chests were opened for lung separation. Three bronchoalveolar lavages (BALs) were performed in the left lung using $-4^{\circ} \mathrm{C}$ normal saline (Tamaoki et al., 1995), and the BAL fluid (BALF) was centrifuged at $1000 \mathrm{rpm}$ for $10 \mathrm{~min}$. The right upper lung tissues were dissected into $1 \times 1 \mathrm{~mm}$ pieces, and then immersed in TRIzol Reagent solution (Invitrogen, Carlsbad, CA, USA) for the determination of HMGB 1 mRNA and protein expression. The right middle lung tissues were fixed with $10 \%$ formalin, embedded in paraffin, and stained with hematoxylin and eosin (HE) for the observation of pathological changes; the scores were calculated via Smith grading (Smith et al., 1997). Right lower lung tissues were fixed in $2.5 \%$ glutaraldehyde solution for the observation of ultra-structural changes under a transmission electron microscope (TEM).

\section{Real-time (quantitative) reverse transcription polymerase chain reaction (qRT-PCR)}

The total RNA was extracted from right upper lung tissues using TRIzol Reagent and $1 \mu \mathrm{g}$ RNA was reverse transcribed to complementary DNA (cDNA) with random hexamer primers and MMLV reverse transcriptase (Fermentas, Vilnius, Lithuania). Real-time PCR was performed using a SYBR Green mix (Takara, Madison, WI, USA) on the ABI 7500 cycler (Applied Biosystems, Carlsbad, CA, USA). PCR primers used were as follows: $H M G B 1$, sense 5'-GAG GTA GGG CAT GAG GAT GA-3', antisense 5'-CCT TCA TCA CCG GTT TCT 
GT-3'; $\beta$-actin, sense 5'-GTA GCC ATC CAG GCT GTG TT-3', antisense 5'-CCC TCA TAG ATG GGC ACA GT-3'. The amplification was optimized using the following PCR program: $95^{\circ} \mathrm{C}$ for $5 \mathrm{~min}$, followed by 40 cycles of denaturation at $95^{\circ} \mathrm{C}$ for $15 \mathrm{~s}$ and annealing at $60^{\circ} \mathrm{C}$ for $45 \mathrm{~s}$. Differences in gene expression between groups were calculated using cycle threshold $(\mathrm{Ct})$ values, which were normalized against $\beta$-actin transcript levels and expressed as values relative to the control.

\section{Western blotting}

The right upper lung tissues were lysed in RIPA lysis buffer and the total protein was quantified by bicinchoninic acid protein assay kit (BI Yuntian Co, Shanghai, China) according to manufacturer instructions. Next, $20 \mu \mathrm{g}$ protein was separated by sodium dodecyl sulfatepolyacrylamide gel electrophoresis and transferred to a nitrocellulose membrane. After blocking with skimmed milk overnight for $1 \mathrm{~h}$, membranes were incubated with an anti-HMGB1 polyclonal antibody (Abcam, Cambridge, MA, 1:1000 dilution) and an anti- $\beta$-actin polyclonal antibody (CST, Danvers, MA, USA, 1:1000 dilution) overnight at $4^{\circ} \mathrm{C}$, followed by horseradish peroxidase-conjugated secondary antibody $(1: 10000)$ for $1 \mathrm{~h}$. The blots were visualized by enhanced chemiluminescence reagents (Millipore, Tokyo, Japan).

\section{Enzyme-linked immunosorbent assay}

Contents of HMGB1, TNF- $\alpha$, and IL- 6 in the blood and BALF were detected using sandwich-type enzyme-linked immunosorbent assay kits (R\&D Systems; Minneapolis, MO, USA) according to manufacturer instructions.

\section{Statistical analysis}

Measurement data are reported as means \pm standard deviation (SD), and analyzed using the SPSS18.0 software (SPSS, Chicago, IL, USA). Comparison between groups was conducted via the Student $t$-test and one-way analysis of variance. Furthermore, comparison of the enumeration data was performed via the $\chi^{2}$ test. $\mathrm{P}<0.05$ was considered to be statistically significant.

\section{RESULTS}

\section{Survival rate}

As shown in Figure 1, the CLP + UTI group showed a higher seven-day survival rate than the CLP group $(\mathrm{P}<0.05)$.

\section{Pathological changes and the Smith score}

No pathological changes occurred in the control group, while there were heavier injuries in the CLP group, in which edema was obvious, inflammation and hemorrhage rose in the pulmonary alveolus and mesenchyme, and local atelectasis was observed at $24 \mathrm{~h}$ after the surgery (Figure 2). For the CLP + UTI group, the injury peaked at $24 \mathrm{~h}$ after surgery, but the Smith score was remarkably lower than that in the CLP group at $48 \mathrm{~h}$ after surgery $(\mathrm{P}<0.05)$. 
The CLP group had a higher Smith score than the control group, and the treatment with UTI decreased the scores, but these were still higher than those of control group (Figure 2).

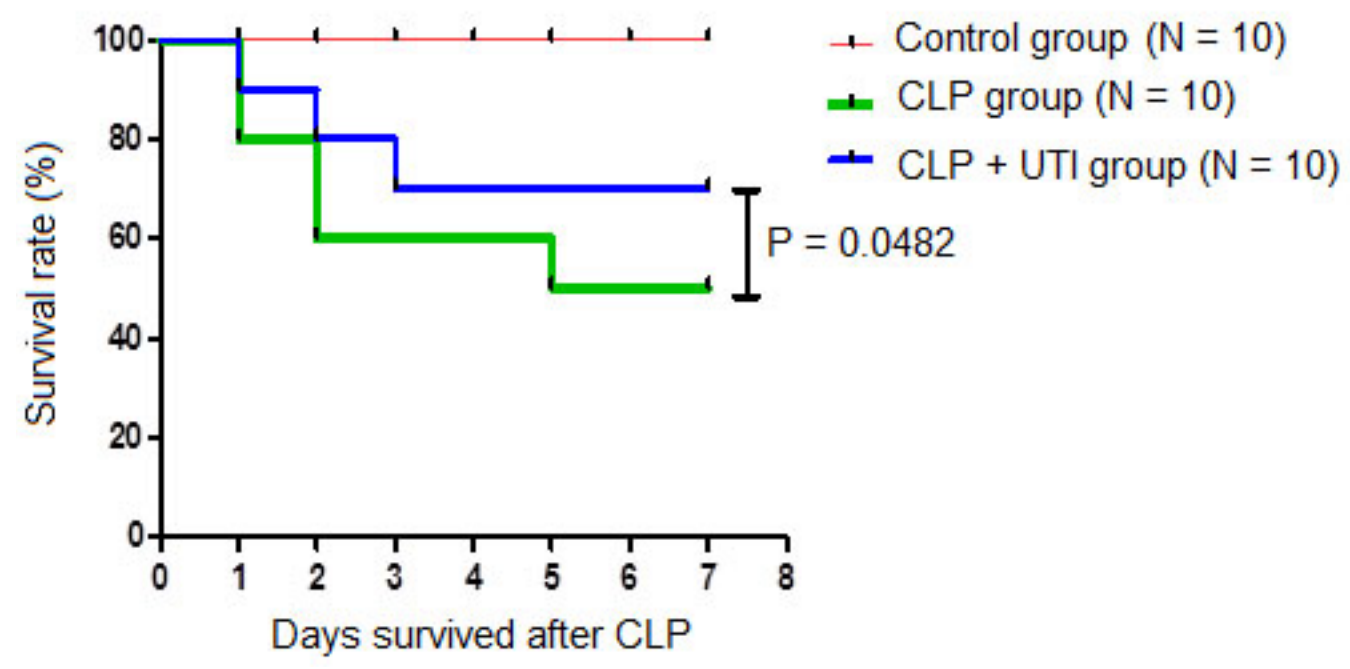

Figure 1. Seven-day survival rate of rats in different groups. CLP $=$ cecal ligation and puncture; UTI = ulinastatin.
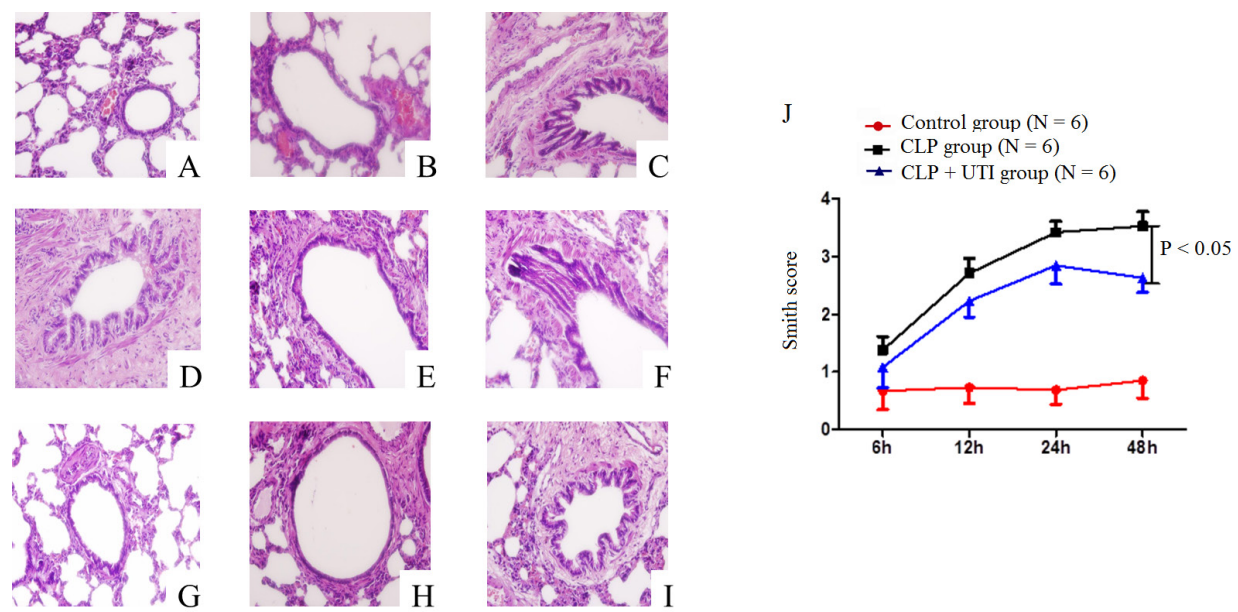

Figure 2. Pathological changes of lung tissues in the three groups (HE stain, 200X). Left: A) control; B) CLP, 6 h; C) CLP, 12 h; D) CLP, 24 h; E) CLP, 48 h; F) CLP + UTI, 6 h; G) CLP + UTI, 12 h; H) CLP + UTI, 24 h; I) CLP + UTI, 48 h; Right: J) the Smith score for each group. CLP = cecal ligation and perforation; UTI = ulinastatin.

\section{Ultra-structural changes of lung tissues}

As observed via electron microscope, cells were closely connected in the control group, which showed normal numbers and the morphology of alveolar epithelium, as well as the regular membrane structure of alveolar capillary membrane. Cells in the CLP group became less connected at $6 \mathrm{~h}$ and $12 \mathrm{~h}$, with decreased organelles and numbers of alveolar 
epithelium; they became worse at $24 \mathrm{~h}$ and $48 \mathrm{~h}$ with cloudy cytoplasm and the stripping of endothelial cells from the alveolar capillary. The lung tissues showed different injuries in the CLP + UTI group at different time points, but the injuries were less severe than those in the CLP group (Figure 3).
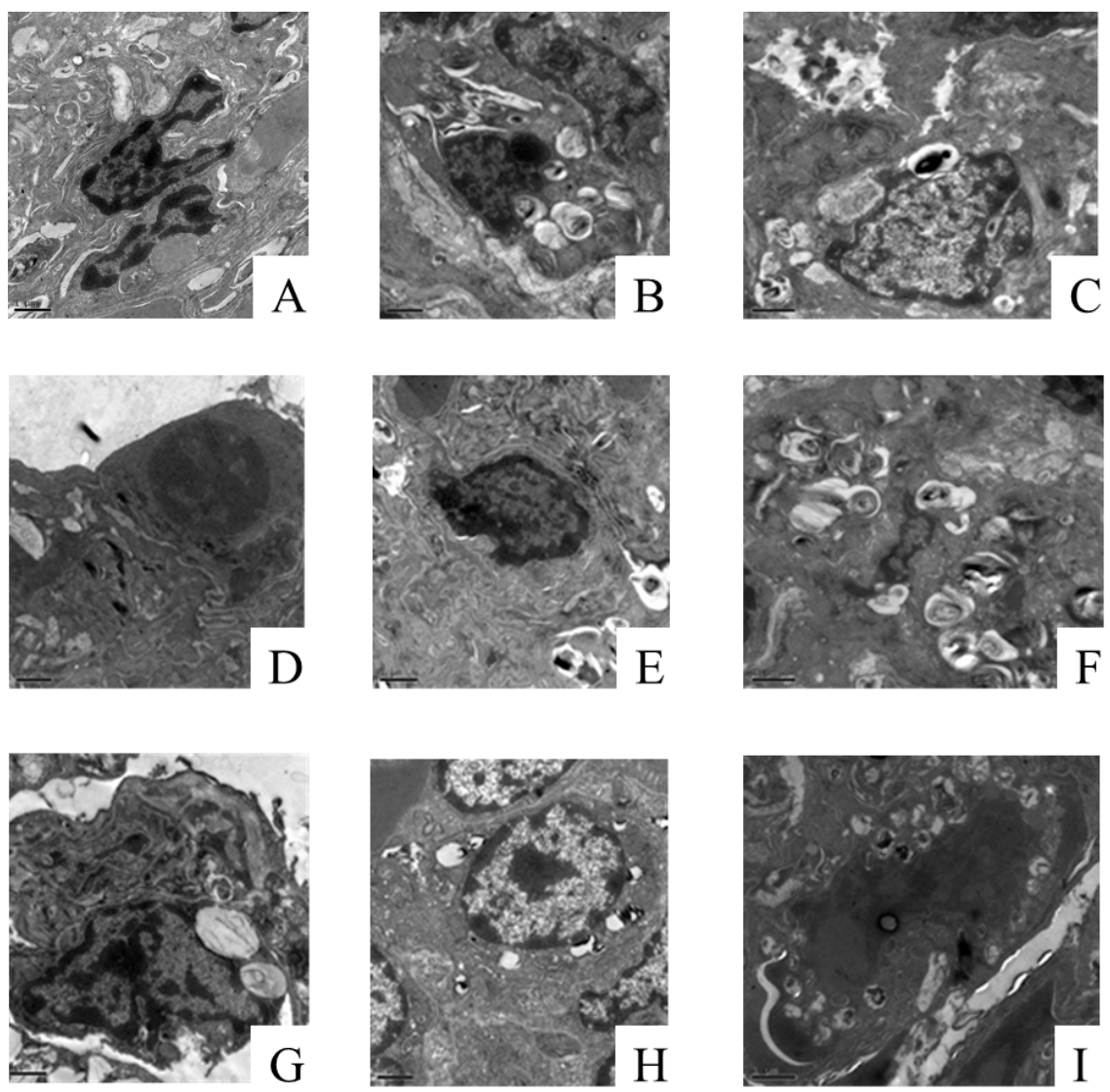

Figure 3. Ultra-structural changes of lung tissues in the three groups.A) control; B) CLP, 6 h; C) CLP, 12 h; D) CLP, $24 \mathrm{~h}$; E) CLP, 48 h; F) CLP + UTI, 6 h; G) CLP + UTI, 12 h; H) CLP + UTI, 24 h; I) CLP + UTI, 48 h. CLP = cecal ligation and perforation; UTI = ulinastatin.

\section{HMGB1 expression changes}

HMGB1 expression was detected in the lung tissues, serum, and BALF. As expected, compared with the control group, the other two groups showed increased HMGB1 expression in the lung tissues, serum, and BALF. However, UTI treatment decreased HMGB1 levels remarkably. Notably, the CLP + UTI group showed significantly lower HMGB1 mRNA expression only at three time points $(12,24$, and $48 \mathrm{~h})$ compared with the CLP group $(\mathrm{P}<0.05)$, but the HMGB1 protein content in the lung tissues, serum, and BALF was significantly reduced after UTI treatment at every time point $(\mathrm{P}<0.05)$ (Figure 4$)$. 

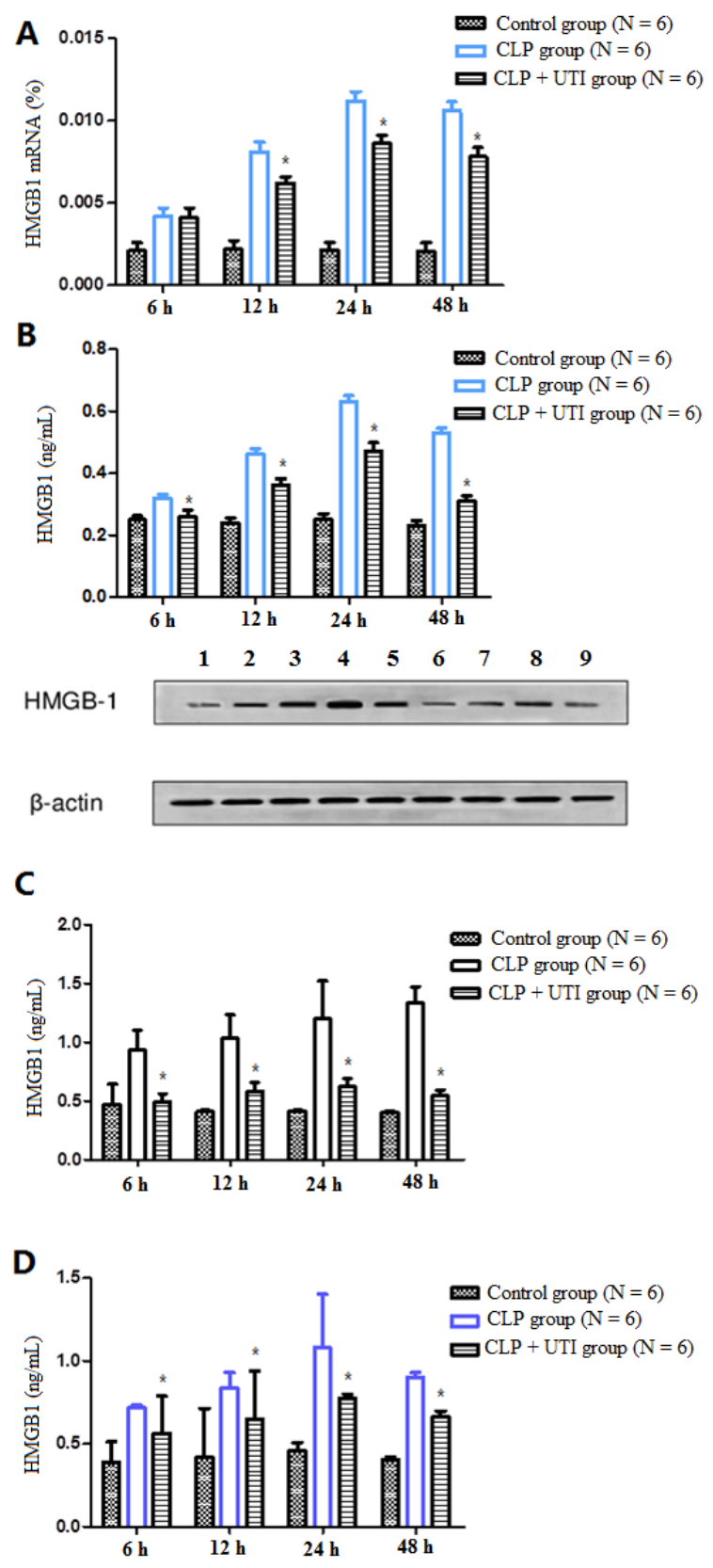

Figure 4. HMGB1 expression in lung tissues of the three groups. A. Relative mRNA expression determined by qRT-PCR; B. protein levels determined by Western blotting. Lane 1: control; Lane 2: CLP, 6 h; Lane 3: CLP, 12 h; Lane 4: CLP, 24 h; Lane 5: CLP, 48 h; Lane 6: CLP + UTI, 6 h; Lane 7: CLP + UTI, 12h; Lane 8: CLP + UTI, 24 h; Lane 9: CLP + UTI, 48 h; C. Serum levels determined by ELISA; D. BALF levels determined by ELISA. $* \mathrm{P}<0.05$ compared with the CLP group. qRT-PCR = reverse transcription real time (quantitative) polymerase chain reaction; HMGB1 = High mobility group box 1; CLP = cecal ligation and perforation; UTI = ulinastatin. 


\section{Changes in TNF- $\alpha$ and IL-6 levels}

The levels of TNF- $\alpha$ and IL-6 changed similarly in serum and BALF. As shown in Figure 5, no significant changes of TNF- $\alpha$ and IL-6 were observed in the control group at different time points, but their levels were significantly lower than those in the other two groups $(\mathrm{P}<$ 0.05). Compared with the CLP group, UTI treatment caused a decrease in levels of TNF- $\alpha$ at 24 $\mathrm{h}(\mathrm{P}=0.022$ for serum; $\mathrm{P}=0.026$ for BALF), and of IL-6 at $24 \mathrm{~h}$ and $48 \mathrm{~h}(\mathrm{P}<0.05)$ (Figure 5).

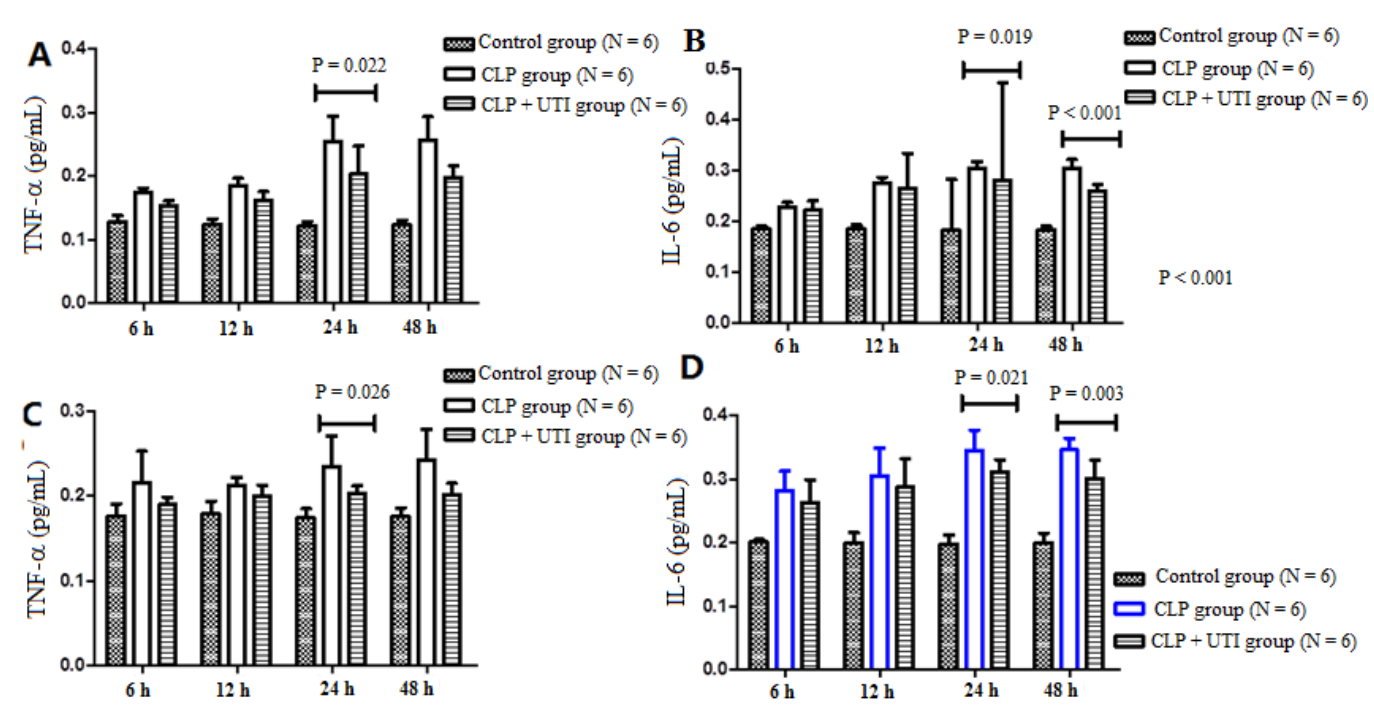

Figure 5. Level changes of TNF- $\alpha$ and IL-6 inthe blood (A and $\mathbf{B})$ and in BALF (C and $\mathbf{D})$. BALF = bronchoalveolar lavage fluid; TNF- $\alpha=$ tumor necrosis factor alpha; IL-6, interleukin6, CLP = cecal ligation and perforation; UTI = ulinastatin.

\section{DISCUSSION}

In this study, we evaluated the protective effects of UTI in a model of CLP-induced ALI in rats. Consistent with previous studies (Fang et al., 2011; Wang et al., 2013), UTI administration improved survival rate and attenuated lung inflammatory injury after CLP, as revealed by decreased hemorrhage, edema, and inflammatory cell infiltration, as well as expression of TNF- $\alpha$ and IL-6. In addition, we also evaluated the lung tissue injury by semiquantitative Smith scoring (Smith et al., 1997) and TEM analysis. As expected, the Smith score was significantly lower in the UTI-treated group compared with CLP alone. TEM analysis indicated the UTI treatment stimulated repair of the alveolar epithelial and endothelial cells that are essential for maintaining the normal alveolar barrier (Geiser, 2003).

TNF- $\alpha$ and IL- 6 in the blood and BALF peaked at $24 \mathrm{~h}$ and $48 \mathrm{~h}$ in the CLP group, but in the CLP + UTI group, the TNF- $\alpha$ levels were significantly lower at $24 \mathrm{~h}$, while the IL-6 levels were lower at both $24 \mathrm{~h}$ and $48 \mathrm{~h}$ than in the CLP group. These findings suggested that UTI might exert a long-term inhibition effect on ALI by downregulating expression of IL-6, but not of TNF- $\alpha$. IL-6 is a multifunctional $26-\mathrm{kDa}$ cytokine responsible for the acute response to infections or systemic inflammation, which then regulate downstream inflammatory mediators 
to fine-tune the overall inflammatory response to environmental triggers (McLoughlin et al., 2005). IL-6 was shown to be increased in the serum and BALF of patients with ALI and high levels are associated with adverse outcomes (Meduri et al., 1995; Parsons et al., 2005). These studies collectively underline the therapeutic potential of targeting IL-6 as a strategy for the management of ALI.

UTI treatment decreased HMGB1 expression at both the mRNA and protein levels in the lung tissue, serum, and BALF. HMGB1 belongs to the subfamily HMGB of the HMG family, the biological activity of which is achieved by interaction with the corresponding receptors, such as the receptor for advanced glycation end products (RAGE) and Toll-like receptors (TLRs) (Pietkiewicz et al., 2007). Deng et al. (2013) reported that the administration of recombination human HMGB1 could induce ALI in rats, and increase the expression of TLR4 in lung tissues, as well as the levels of TNF- $\alpha$ and IL-1 in BALF. Nativel et al. (2013) also demonstrated that recombinant HMGB1 boosted IL-6 expression by RAGE in the inflammation process. Thus, downregulation of HMGB1 expression prevented pulmonary levels of pro-inflammatory cytokines (such as TNF- $\alpha$ and IL-6) and relieved lung injury (Kim et al., 2005; Luan et al., 2013), which was also confirmed by the results following our treatment with UTI. Thus, we believe that UTI might inhibit ALI, as does hepatic ischemia reperfusion injury (Tong et al, 2014), by downregulating HMGB1 leading to lowered TNF- $\alpha$ and IL-6 expression.

There are several limitations to this study. First, this study used a rat model of ALI, which might not absolutely reflect the process occurring in humans; second, we only evaluated the short-term effect of UTI preconditioning on the survival rate, not the long-term efficacy. Therefore, further studies are still needed to clarify the utility of UTI treatment in humans and over the long term.

\section{ACKNOWLEDGMENTS}

Research supported by the Natural Science Foundations of China (\#81171844).

\section{REFERENCES}

Cao YZ, Tu YY, Chen X, Wang BL, et al. (2012). Protective effect of Ulinastatin against murine models of sepsis: Inhibition of TNF- $\alpha$ and IL-6 and augmentation of IL-10 and IL-13. Exp. Toxicol. Pathol. 64: 543-547.

Deng Y, Yang Z, Gao Y, Xu H, et al. (2013). Toll-like receptor 4 mediates acute lung injury induced by high mobility group box-1. PLoS One 8: e64375.

Entezari M, Javdan M, Antoine DJ, Morrow DM, et al. (2014). Inhibition of extracellular HMGB1 attenuates hyperoxiainduced inflammatory acute lung injury. Redox Biol. 2: 314-322.

Fang Y, Xu P, Gu C, Wang Y, et al. (2011). Ulinastatin improves pulmonary function in severe burn-induced acute lung injury by attenuating inflammatory response. J. Trauma 71: 1297-1304.

Geiser T (2003). Mechanisms of alveolar epithelial repair in acute lung injury-a translational approach. Swiss Med. Wkly. 133: 586-590.

Hidaka S, Iwasaka H, Hagiwara S and Noguchi T (2011). Gabexate mesilate inhibits the expression of HMGB1 in lipopolysaccharide-induced acute lung injury. J. Surg. Res. 165: 142-150.

Hochstrasser K, Schönberger OL, Rossmanith I and Wachter E (1981). Kunitz-type proteinase inhibitors derived by limited proteolysis of the inter- $\alpha$-trypsin inhibitor, V. Attachments of carbohydrates in the human urinary trypsin inhibitor isolated by affinity chromatography. Hoppe Seylers Z. Physiol. Chem. 362: 1357-1362.

Ito K, Mizutani A, Kira S, Mori M, et al. (2005). Effect of ulinastatin, a human urinary trypsin inhibitor, on the oleic acidinduced acute lung injury in rats via the inhibition of activated leukocytes. Injury 36: 387-394.

Kim JY, Park JS, Strassheim D, Douglas I, et al. (2005). HMGB1 contributes to the development of acute lung injury after hemorrhage. Am. J. Physiol.-Lung Cell Mol. Physiol. 288: L958-L965. 
Luan ZG, Zhang XJ, Yin XH, Ma XC, et al. (2013). Downregulation of HMGB1 protects against the development of acute lung injury after severe acute pancreatitis. Immunobiology 218: 1261-1270.

McLoughlin RM, Jenkins BJ, Grail D, Williams AS, et al. (2005). IL-6 trans-signaling via STAT3 directs T cell infiltration in acute inflammation. Proc. Natl. Acad. Sci. U. S. A. 102: 9589-9594.

Meduri GU, Headley S, Kohler G, Stentz F, et al. (1995). Persistent elevation of inflammatory cytokines predicts a poor outcome in ARDS plasma IL-1 $\beta$ and IL-6 levels are consistent and efficient predictors of outcome over time. Chest 107: 1062-1073.

Nativel B, Marimoutou M, Thon-Hon VG, Gunasekaran MK, et al. (2013). Soluble HMGB1 is a novel adipokine stimulating IL-6 secretion through RAGE receptor in SW872 preadipocyte cell line: contribution to chronic inflammation in fat tissue. PLoS One 8: e76039.

Nishiyama T, Aibiki M and Hanaoka K (1996). The effect of ulinastatin, a human protease inhibitor, on the transfusioninduced increase of plasma polymorphonuclear granulocyte elastase. Anesth. Analg. 82: 108-112.

Parsons PE, Eisner MD, Thompson BT, Matthay MA, et al. (2005). Lower tidal volume ventilation and plasma cytokine markers of inflammation in patients with acute lung injury. Crit. Care Med. 33: 1-6.

Pietkiewicz J, Seweryn E, Bartyś A and Gamian A (2007). Receptors for advanced glycation end products and their physiological and clinical significance. Postepy Hig. Med. Dosw. 62: 511-523.

Razavi HM, Werhun R, Scott JA, Weicker S, et al. (2002). Effects of inhaled nitric oxide in a mouse model of sepsisinduced acute lung injury. Crit. Care Med. 30: 868-873.

Rubenfeld GD, Caldwell E, Peabody E, Weaver J, et al. (2005). Incidence and outcomes of acute lung injury. N. Engl. J. Med. 353: 1685-1693.

Shen J, Gan Z, Zhao J, Zhang L, et al. (2012). Ulinastatin reduces pathogenesis of phosgene-induced acute lung injury in rats. Toxicol. Ind. Health. 30: 785-793

Silva E, Arcaroli J, He Q, Svetkauskaite D, et al. (2007). HMGB1 and LPS induce distinct patterns of gene expression and activation in neutrophils from patients with sepsis-induced acute lung injury. Intensive Care Med.. 33: 1829-1839.

Smith KM, Mrozek JD, Simonton SC, Bing DR, et al. (1997). Prolonged partial liquid ventilation using conventional and high-frequency ventilatory techniques: gas exchange and lung pathology in an animal model of respiratory distress syndrome. Crit.Care Med. 25: 1888-1897.

Sumi H, Takada Y and Takada A (1977). Studies on human urinary trypsin inhibitor 1. Its modification on treatment of urine with acid. Thromb. Res. 11: 747-754.

Tamaoki J, Tagaya E, Yamawaki I, Sakai N, et al. (1995). Effect of erythromycin on endotoxin-induced microvascular leakage in the rat trachea and lungs. Am. J. Respir. Crit. Care Med. 151: 1582-1588.

Tong Y, Tang Z, Yang T, Yang Y, et al. (2014). Ulinastatin preconditioning attenuates inflammatory reaction of hepatic ischemia reperfusion injury in rats via high mobility group box 1 (HMGB1) inhibition. Int. J. Med. Sci. 11: 337-43.

Wang N, Liu X, Zheng X, Cao H, et al. (2013). Ulinastatin is a novel candidate drug for sepsis and secondary acute lung injury, evidence from an optimized CLP rat model. Int. Immunopharmacol. 17: 799-807.

Ware LB and Matthay MA (2000). The acute respiratory distress syndrome. N. Engl. J. Med. 342: 1334-1349. 